\title{
Synthesis and characterization of high performance superabsorbent hydrogels using bis[2-(methacryloyloxy)ethyl] phosphate as crosslinker
}

\author{
A. A. L. Gonçalves, A. C. Fonseca, I. G. P. Fabela, J. F. J. Coelho, A. C. Serra* \\ CEMUC, Department of Chemical Engineering, University of Coimbra, Rua Sílvio Lima-Pólo II, 3030-290, Coimbra, \\ Portugal
}

Received 13 August 2015; accepted in revised form 13 October 2015

\begin{abstract}
Various superabsorbent polymers (SAPs) were synthesized by free radical copolymerization at $70^{\circ} \mathrm{C}$ using acrylic acid (AA), potassium acrylate (KA), $N$-isopropyl acrylamide (NIPAM) and sulfopropyl methacrylate potassium salt (SPM) as monomers, bis[2-(methacryloyloxy)ethyl] phosphate (BMEP) as crosslinker and potassium persulfate (KPS) as initiator. The optimization of the synthesis led to the preparation of a SAP with very high water absorption ability, with a maximum swelling of $2618 \mathrm{~g}$ water/g dry hydrogel. The most promising SAP was fully characterized and the absorption capacities were studied at different $\mathrm{pH}$ and ionic strengths. When this SAP was mixed with soil, the mixture was able to lose water more slowly. Also, this material revealed high loading capacity and showed good releasing profiles using urea as model fertilizer. Due to these advantageous properties, the synthesized SAP can be used in agricultural applications.
\end{abstract}

Keywords: polymer gels, superabsorbent, bis[2-(methacryloyloxy)ethyl] phosphate, agriculture

\section{Introduction}

Superabsorbent polymers (SAPs) are a class of hydrogel like materials with increasing interest in agriculture or in polluted lands [1]. These 3D structures can absorb large amounts of water or aqueous fluids in relatively short periods of time, as compared to conventional hydrogels, and generally absorb an amount of water that can reach 1000 times (or more) their dry weight material $[2,3]$. Hydrogels can be applied in a wide range of products and areas (e.g., hygiene, biomedical, pharmaceutical, agriculture) due to their unique properties such as hydrophilicity, biocompatibility and especially high absorption capacity and swelling/de-swelling behaviour [3-5]. Particularly, SAPs appear as materials with promising characteristics to improve the use of water in soils. These materials can maximize water availability and increase crop production without adverse consequences for the natural resources and environment. It is known that the largest consumers of fresh water are the agricultural activities, specifically intensively irrigated agriculture, which currently consumes over $85 \%$ of the available water. The importance of this comes from the fact that a very high fraction of produced food comes from irrigated lands $[6,7]$. Also, the increased water demand in several sectors like industry and domestic, among others, associated to the irregular distribution due to climatic and environmental changes and the practice of some inefficient methods, demands an urgent development of effective technologies to rationalize the use of water. Another important feature that can be explored in SAPs is their capacity to release agrochemicals in a controlled way. This characteristic proves to be quite useful and advantageous for a precise supply of nutrients to plants. Among other

\footnotetext{
${ }^{*}$ Corresponding author, e-mail: aserra@eq.uc.pt

(C) BME-PT
} 
aspects, it allows the correction of environmental deficiencies, avoiding additional costs due to excessive fertilization and agrochemical wastes [8], reducing water runoff and erosion, enabling the recovery of desertified and slightly fertile areas and others $[7,9]$.

The present work involved the synthesis of hydrogels with extremely high swelling properties and a promising potential for use in agricultural applications, either as water reservoirs or as agrochemical release systems. The synthesis of these SAPs was carried out using a combination of monomers, such as AA, KA, NIPAM and SPM. To the best of our knowledge, BMEP has never been used as a crosslinker for the synthesis of hydrogels. The swelling properties of all SAPs were evaluated. For the most promising SAP, the ability to incorporate and release urea (used as fertilizer model compound) and the improvements in water retention capacity by soil were studied.

\section{Experimental section}

\subsection{Materials}

Acrylic acid (AA) (99\%), 3-sulfopropyl methacrylate potassium salt (98\%) (SPM), bis[2-(methacryloyloxy)ethyl] phosphate (99.9\%) (BMEP), potassium hydroxide (KOH) (90\%), hydrochloric acid ( $\mathrm{HCl})(37 \%)$ were purchased from Sigma-Aldrich (St Louis, USA); N-isopropyl acrylamide (98\%) (NIPAM) was purchased from TCI Europe (Zwijndrecht, Belgium); potassium persulfate (KPS) (99\%) was acquired from Riedel-de-Haën (Seelze, Germany). Sodium hydroxide $(\mathrm{NaOH})(97 \%)$ was obtained from Panreac Química (Barcelona, Spain). Urea was acquired from VWR International, Ltd (Carnaxide, Portugal). Potassium acrylate (KA) was prepared by neutralization of acrylic acid with the corresponding amount of base $(\mathrm{KOH})$. All the reagents, solvents and other materials were used as received without further purification.

\subsection{SAP synthesis}

The synthesis of the SAPs was performed in a $150 \mathrm{~mL}$ glass reactor, equipped with a Liebig condenser and a mechanical stirrer. The reagents, AA, KA, SPM, NIPAM, BMEP and KPS were added to the reactor with $40 \mathrm{~mL}$ of distilled water. The mixture was heated at $70^{\circ} \mathrm{C}$ until the gelification of the mixture occurred (generally between 60 and 75 minutes). After the polymerization, the SAP samples were washed with cold methanol to remove unreacted monomers, cut into small pieces and dried at $50^{\circ} \mathrm{C}$ for $24 \mathrm{~h}$. Next, the dry SAPs were purified by Soxhlet extraction using acetone-methanol $(1: 1 \mathrm{v} / \mathrm{v})$, at $50^{\circ} \mathrm{C}$ for $24 \mathrm{~h}$, powdered, dried until constant weight and stored. For all reactions, the conversion was calculated gravimetrically and was close to $95 \%$.

\subsection{Instrumentation}

The Fourier Transform Infrared (FTIR) spectra were recorded on a FTIR-spectrophotometer (Jasco) with $4 \mathrm{~cm}^{-1}$ resolution. Attenuated Total Reflection (ATR) mode was used. Scanning electron microscope (SEM) (MEV)/EDS, JEOL, model JSM-5310, at an accelerating voltage of $10 \mathrm{kV}$ was used for SEM analysis. In this especific case, for the analysis of porosity, the SAP samples were first immersed in distilled water until reaching equilibrium swelling, placed in liquid nitrogen, then freeze dried and analysed by SEM. TGA was perfomed in a SDT Q600 equipment (TA instruments), at a heating rate of $10^{\circ} \mathrm{C} / \mathrm{min}$, for temperatures ranging from 30 to $600^{\circ} \mathrm{C}$, under a constant nitrogen flow.

\subsection{Molecular weight between crosslinks $\left(M_{\mathrm{c}}\right)$}

The average molecular weight between crosslinks $\left(M_{\mathrm{c}}\right)$ of the hydrogels was calculated from the volume fraction of polymer in the swollen polymer. The polymer volume fraction $(v)$ of the swollen polymer was determined taking into account the swelling capacity of the hydrogels in distilled water and the density of the polymer determined in a pycnometer using heptane, according to Equation (1) [10, 11]:

$\nu=\frac{W_{0} \cdot \rho_{\mathrm{w}}}{W \cdot \rho-W_{0} \cdot\left(\rho-\rho_{\mathrm{w}}\right)}$

where $W$ is weight of swollen polymer, $W_{0}$ is the initial weight of the sample and $\rho_{\mathrm{w}}$ and $\rho$ are the density of water and the density of the dry hydrogel.

The average molecular weight between crosslinks $\left(M_{\mathrm{c}}\right)$ can be estimated using Equation (2) developed by Flory and Rehner [12]:

$$
M_{\mathrm{c}}=-\frac{\rho \cdot V_{1} \cdot \nu^{1 / 3}}{\frac{K \cdot \nu^{2}}{2+\ln (1-\nu)+\nu}}
$$

where $V_{1}$ is the molar volume of water $\left(18.062 \mathrm{~cm}^{3} / \mathrm{mol}\right)$ and $K=2 \cdot X \cdot V_{1} /(R \cdot T)$ [12] is the polymer-solvent interaction parameter and indicates the change of interaction energy when solvent and 
polymer are mixed together. The value of $\chi$ was calculated using Equation (3):

$\chi \cong \frac{1}{2}+\frac{\nu}{3}$

\subsection{Determination of crosslinking density $(q)$}

The determination of the crosslinking density was done through Equation (4) [13]:

$q=\frac{M_{\mathrm{c}}}{M_{\mathrm{r}}}$

where $M_{\mathrm{r}}$, the pondered molar mass of the repeating unit was determined through the Equation (5) [13], where $M_{\mathrm{NIPAM}}, M_{\mathrm{KA}}, M_{\mathrm{SPM}}, M_{\mathrm{AA}}$ are the molar masses and $m_{\mathrm{NIPAAm}}, m_{\mathrm{KA}}, m_{\mathrm{SPM}}$ and $m_{\mathrm{AA}}$ are the amount of the used monomers. paper and the bag was weighted [3]. This process was repeated several times until the swelling equilibrium was reached (approximately 24 hours), i.e. until the bag presented a constant weight. The swelling profiles and the absorption capacity (AC) of the hydrogel in units of amount $[\mathrm{g}]$ of water absorbed per gram of SAP, can be defined using Equation (7):

$\mathrm{AC}=\frac{W_{\mathrm{s}}-W_{\mathrm{d}}}{W_{\mathrm{d}}}$

where $W_{\mathrm{s}}$ and $W_{\mathrm{d}}$ are the weights of the swollen hydrogel and the dry sample, respectively. The final absorption capacity was calculated by taking an average of three independent measurements.

$M_{\mathrm{r}}=\frac{M_{\mathrm{AA}} \cdot m_{\mathrm{AA}}+M_{\mathrm{NIPAM}} \cdot m_{\mathrm{NIPAM}}+M_{\mathrm{KA}} \cdot m_{\mathrm{KA}}+M_{\mathrm{SPM}} \cdot m_{\mathrm{SPM}}}{m_{\mathrm{AA}}+m_{\mathrm{NIPAM}}+m_{\mathrm{KA}}+m_{\mathrm{SPM}}}$

\subsection{Gel content}

To determine the gel content of the SAPs, hydrogel samples were cut and dried at $50^{\circ} \mathrm{C}$ for $24 \mathrm{~h}$. The dried hydrogels were then extracted in a Soxhlet using acetone-methanol $(1: 1 \mathrm{v} / \mathrm{v})$ at $50^{\circ} \mathrm{C}$ for $24 \mathrm{~h}$. The portion of the hydrogel remaining after extraction, corresponding to the insoluble and crosslinked part, was dried and weighed. The gel content was calculated according to Equation (6) [14]:

Gel content $[\%]=\frac{W_{1}}{W_{0}} \cdot 100$

where $W_{1}$ is the weight of the insoluble part of the sample (after the extracion) and $W_{0}$ is the weight of dried hydrogel before extraction.

\subsection{Swelling Properties}

The absorption capacity of the synthesized SAPs was measured both in distilled water and in saline solutions $(0.3,0.6,0.9$ and $1.5 \mathrm{wt} \%$ of $\mathrm{NaCl})$, at various pHs $(3,5,7,9,11)$ using the tea bag method [3].

For the tea bag method, an accurately weighed powdered SAP sample (0.005 and $0.01 \mathrm{~g})$ was placed into a tea-bag (acrylic/polyester gauze with fine meshes) and the bag was immersed in an excess amount of water or of another solution (approximately 1 liter) at room temperature. After a certain period of time, the bag was removed from the solution, the excess of water was removed superficially with tissue

\subsection{Water retention capacity of the soil with SAP}

To study the water retention capacity of the soil treated with the SAP, different amounts of hydrogel $(0.5,1$ and $2 \mathrm{wt} \%$ relative to the soil weight) were mixed with $40 \mathrm{~g}$ of sandy soil. Subsequently, the mixtures were uniformly irrigated with a certain amount of distilled water $(20 \mathrm{~g})$ and exposed to air at a room temperature. For each mixture, three experiments were performed. A control experiment was also performed using soil without addition of hydrogel [15, 16]. These mixtures were weighed at certain intervals of time and the weight loss by water evaporation was registered. To determine the water retention (WR) percentage of the soil with or without the hydrogel, Equation (8) was used [15].

$\mathrm{WR}[\%]=\frac{W_{0}-W_{\mathrm{t}}}{W_{0}} \cdot 100$

where $W_{0}$ and $W_{\mathrm{t}}$ are the initial mixture weight, and the mixture weight at a certain time, respectively.

\subsection{Urea loading and release}

To perform the urea loading tests, samples of dry hydrogel $(0.005 \mathrm{~g})$ were immersed in urea solutions with different concentrations (5, 10, 15 and $20 \mathrm{~g} / \mathrm{L}$ ) for approximately 24 hours (until reaching the maximum swelling) [11, 15]. Subsequently, the swollen and loaded hydrogels were oven dried at $50^{\circ} \mathrm{C}$ to constant weight. Knowing the weights of unloaded 
$\left(M_{0}\right)$ and loaded dry hydrogels $\left(M_{1}\right)$ it was possible to calculate the percentage of loading of urea using Equation (9) [14]:

Loading [\%] $=\frac{M_{1}-M_{0}}{M_{0}} \cdot 100$

For each concentration of urea three experiments were performed and an average of these values was used to carried out the calculations.

To investigate the ability of the SAPs to release urea, three samples of urea loaded hydrogels (obtained from the immersion in a urea solution with a concentration of $20 \mathrm{~g} / \mathrm{L}$ ) with weights between 0.120 and 0.170 grams were placed in $500 \mathrm{~mL}$ of distilled water at room temperature. At various times, $20 \mathrm{~mL}$ of this solution was collected for a glass container and the content was freeze dried. The amount of urea was determined gravimetrically, comparing the weight of the container before and after this process and taking into account a blank experiment with only $20 \mathrm{~mL}$ of water. The percentage of urea released was calculated using the Equation (10) [11]:

$\%$ of urea released $=$

$$
=(\Delta W)_{\mathrm{n}} \cdot \frac{500-(n-1) \cdot 20}{20}+\sum_{\mathrm{i}=1}^{\mathrm{n}-1} \frac{(\Delta W)_{\mathrm{i}}}{W_{\mathrm{u}}}
$$

where $(\Delta W)_{\mathrm{i}}$ is the weight of urea released from the $20 \mathrm{~mL}$ sample, $W_{\mathrm{u}}$ is the amount of urea loaded in SAP, $n$ indicates the number of urea release measurements at various time intervals in a single experiment, $(\Delta W)_{\mathrm{n}}$ is the weight of urea released in the measurement $n$.

\section{Results and discussion}

\subsection{Synthesis and characterization of the SAPs}

The present work describes the synthesis of superabsorbant hydrogels from SPM, AA, NIPAM and KA monomers, which are the common monomers used in the preparation of SAPs, but introducing BMEP, a generally used flame retardant monomer [17], as the difunctional crosslinker. The presence of phosphate groups could bring to the gel higher biocompatibility and biodegradability. SPM was used to increase the osmotic pressure between the interior and the exterior of the hydrogel (due to the difference in the concentration of the $\mathrm{K}^{+}$counterion), as a mean to improve the water absorption capacity of the ionic hydrogel [18]. The use of this crosslinker in combination with the above refered monomers has never been reported in hydrogel synthesis. NIPAM is known to increase the rate of decomposition of persulfates, and because of that it was used in all the hydrogels prepared in this work $[11,19,20]$. The different reaction conditions for SAP synthesis (SAP1 to SAP10) and the physical properties of the obtained products are presented in Table 1.

\subsection{Mechanism of polymerization}

The synthesis of SAPs occurred through free radical polymerization as shown in Figure 1. The initiator decomposed thermally to produce sulphate radicals that add to monomers, propagating a chain reaction that produces the crosslinked polymer (SAP) [21].

\subsection{Swelling properties}

The results for the water absorption capacity of the different synthesized SAPs (Table 1) shows that these

Table 1. Monomer feed composition, average molecular weight between crosslinks $\left(M_{\mathrm{c}}\right)$, crosslinking density $(q)$, gel content and absorption capacity (AC) of synthesized SAPs

\begin{tabular}{|l|c|c|c|c|c|c|c|c|}
\hline \multicolumn{1}{|c|}{ SAP } & $\begin{array}{c}{[\mathbf{A A}]} \\
{[\mathbf{M}]}\end{array}$ & $\begin{array}{c}{[\mathbf{K A}]} \\
{[\mathbf{M}]}\end{array}$ & $\begin{array}{c}{[\mathbf{S P M}]} \\
{[\mathbf{M}]}\end{array}$ & $\begin{array}{c}{[\mathbf{B M E P}]} \\
{[\mathbf{M}]}\end{array}$ & $\begin{array}{c}\boldsymbol{M}_{\mathbf{c}} \\
{[\mathbf{g} / \mathbf{m o l}]}\end{array}$ & $\boldsymbol{q}$ & $\begin{array}{c}\text { Gel content } \\
{[\%]}\end{array}$ & $\begin{array}{c}\text { AC } \\
{\left[\mathbf{g}_{\text {water }} / \mathbf{g}_{\text {dry hydrogel }}\right]}\end{array}$ \\
\hline SAP1 & 0.635 & 0.715 & 0.635 & 0.00118 & 14093600 & 78997 & 86 & 1387 \\
\hline SAP2 & 1.270 & 0.358 & 0.358 & 0.00118 & 31511864 & 212717 & 92 & 1715 \\
\hline SAP3 & - & 0.715 & 1.270 & 0.00118 & 26630539 & 122481 & 93 & 1521 \\
\hline SAP4 & 0.953 & 0.715 & 0.318 & 0.00118 & 11928455 & 82386 & 87 & 1175 \\
\hline SAP5 & 1.270 & - & 0.715 & 0.00118 & 24793604 & 133867 & 88 & 2618 \\
\hline SAP6 & 1.270 & 0.538 & 0.178 & 0.00118 & 23575791 & 192227 & 85 & 1469 \\
\hline SAP7 & - & - & 1.985 & 0.00118 & 14036977 & 57341 & 92 & 1587 \\
\hline SAP8 & 1.270 & - & 0.715 & 0.00155 & 25217194 & 139719 & 91 & 1696 \\
\hline SAP9 & 1.270 & - & 0.715 & 0.00195 & 25877562 & 136154 & 93 & 1484 \\
\hline SAP10 & 1.270 & - & 0.715 & 0.00233 & 26093318 & 140884 & 90 & 1376 \\
\hline
\end{tabular}

Constant amounts of NIPAM $(0,05 \mathrm{M})$ and KPS $(0,009 \mathrm{M})$ were used in all experiments for a polymerization volume of $40 \mathrm{~mL}$; AA (acrylic acid); KA (potassium acrylate); SPM (3-sulfopropyl methacrylate potassium salt.); BMEP (bis[2-(methacryloyloxy)ethyl] phosphate). AC corresponds to the absorption capacity in distilled water. 

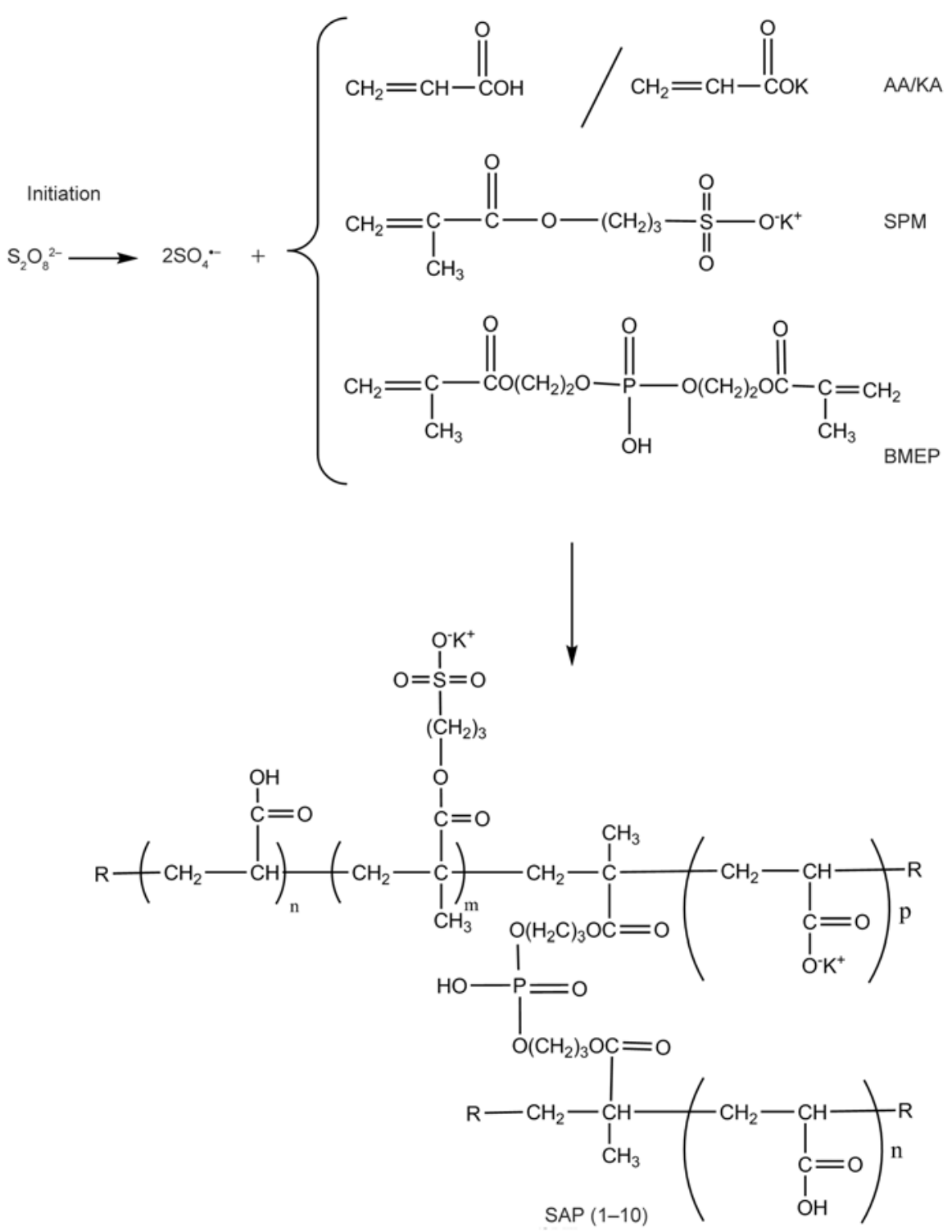

Figure 1. Reaction scheme to originate SAP polymers

materials have, in general, excellent swelling properties with very high water absorption capacities between 1175 and $2618 \mathrm{~g}$ water/g dry hydrogel. This fact is explained by the high charge density of the material, and it is particularly relevant in the case of SAP5. From Table 1, it is possible to observe that the gel contents are between $80-90 \%$, suggesting that during the reaction there was an efficient formation of an insoluble copolymer network and that the synthesized hydrogels have a highly crosslinked structure.

The approximate molecular weight between crosslinks, obtained from Equation (2), showed high values for the different SAPs, between 11.928 and $31.511 \mathrm{Mg} / \mathrm{mol}$. Also, the polymers showed a high crosslinking density (60 788 to 221063 ). Unfortunately, no direct correlation between values of $M_{\mathrm{c}}$ and $q$ with polymer swelling capacity can be made. The same was observed in the work of Venkatachalam et al. [11]. In the case of SAP5, which showed the highest swelling capacity value, the molecular weight between crosslinkings was approximately 24.793 millions with a $q$ value of 140724 . Comparing with related hydrogels [11] using trimethylolpropane as crosslinking agent, the use of BMEP for the same purpose and SPM as a major monomer greatly improved the swelling properties of the materials.

The water absorption capacity of the SAPs is influenced by the nature and relative amounts of monomers used in the synthesis. The maximum water absorption capacity of SAPs with different amounts of monomers (SAP1-SAP7) are represented in Figure 2. 


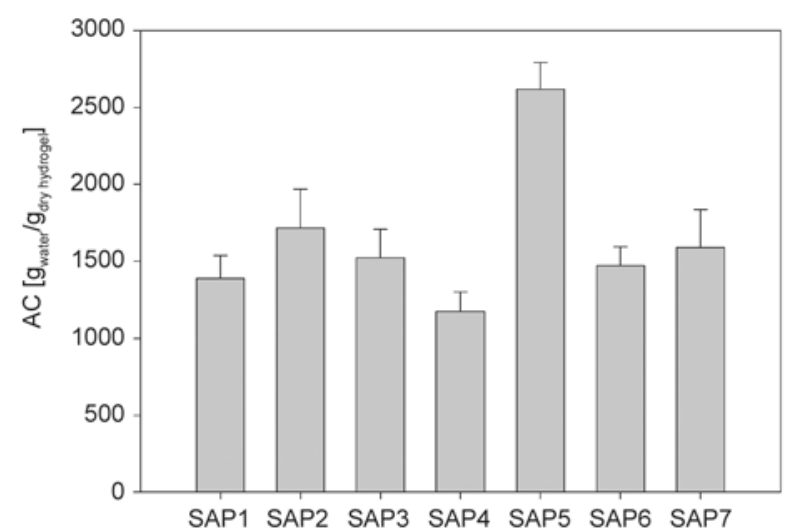

Figure 2. Water absorption capacity for the different SAP

The results shown in Figure 2 indicate that the water absorption capacity is higher for the hydrogels containing higher amounts of two monomers, AA and SPM (SAP5). When the amount of SPM decreases and $\mathrm{KA}$ is used as co-monomer $(0.358 \mathrm{KA}$ and 0.358 SPM on SAP2 or 0.178 SPM and $0.538 \mathrm{KA}$ for SAP6), hydrogels with lower absorption capacities are obtained. The presence of AA is essential to afford high swelling properties. Hydrogels prepared in the absence of this monomer (SAP7 and SAP3) originate materials with lower swelling values. It is assumed that the presence of the highly polar sulfonate group in the structure of the SPM has a key role in the absorption capacity of the SAPs $[22,23]$. The amount of crosslinker is also determinant for the swelling behaviour of hydrogels [4, 24]. The most promising result was obtained for SAP5, where AA and SPM were used as monomers, and with a BMEP concentration of $0,00118 \mathrm{M}$. The ratio of charged monomers to BMEP is very low (approximately $1.100)$ as well as its concentration $(0.0018 \mathrm{M})$. These two parameters contribute to low crosslink density in the gel structure which favours swelling properties. Attempts were made to synthesize SAPs with crosslinker concentrations lower than $0,00118 \mathrm{M}$, but the obtained material dissolved in water when swelling studies were carried out. At low concentration of crosslinking agent, there is not enough crosslinking density and the formed network is too loose and does not have enough strength to hold water molecules inside the structure $[4,25]$. The concentration initially used seems to provide an interesting compromise between the crosslinking level and a stable gel structure. Increasing the amount of BMEP decreases the water absorption capacities (Figure 3). At crosslinker concentrations higher than $0,00118 \mathrm{M}$, hydrogels with higher crosslinking densities were

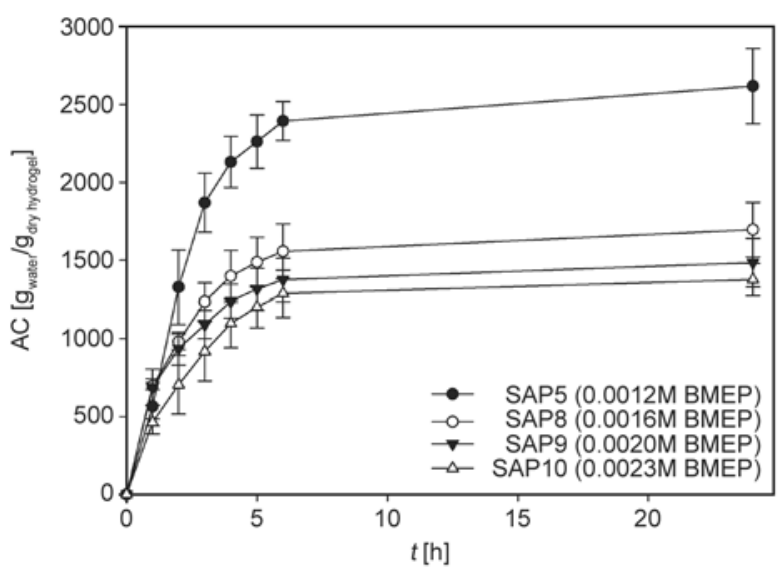

Figure 3. Effect of crosslinker amount (BMEP) on water absorption capacity of SAPs

obtained, leading to lower water absorption capacities. In these conditions, a tighter network structure, with a high number of crosslinking points is expected, which results in a reduction in the absorption capacity of the hydrogel [9].

The swelling profiles in distilled water (Figure 3) also shows that all SAPs present a fast water absorption capacity. SAP5, with higher swelling capacity, presents almost the same initial water absorption capacity as the other SAPs but mantains this initial absorption rate for a longer time $(7 \mathrm{~h})$ unlike the others, that at this time, presents a much lower water absorption value. Interestingly the time to achieved almost the maximum swelling values is the same for all the SAPs $(7 \mathrm{~h})$.

Due to the excellent results obtained for SAP5, in terms of water swelling and also in terms of reproducibility (two synthesis carried out at different times led to SAPs with swelling abilities of 2437 and $\left.2618 \mathrm{~g}_{\text {water }} / \mathrm{g}_{\text {dry hydrogel }}\right)$, it was decided to fully characterize only this SAP in terms of chemical structure, morphology, and thermal stability. Its swelling capacity in solutions with different ionic strengths, and different pHs was also studied. Finally, this SAP was tested as a release system using urea as model compound.

\subsection{SAP5 characterization}

The FTIR spectra of SAP5, AA and SPM are presented in Figure 4.

From Figure 4a, it is possible to observe the disappearance of the bands corresponding to the double bonds $\left(v-\mathrm{C}=\mathrm{C}-\right.$ at $c a .1636 \mathrm{~cm}^{-1}$ and $\delta-\mathrm{C}=\mathrm{C}-$ at $c a$. $815 \mathrm{~cm}^{-1}$ ) in the spectrum of SAP5. In Figure $4 \mathrm{~b}$ only the spectrum of SAP5 is represented. It is possible to identify a band at $c a .1720 \mathrm{~cm}^{-1}$ that has con- 


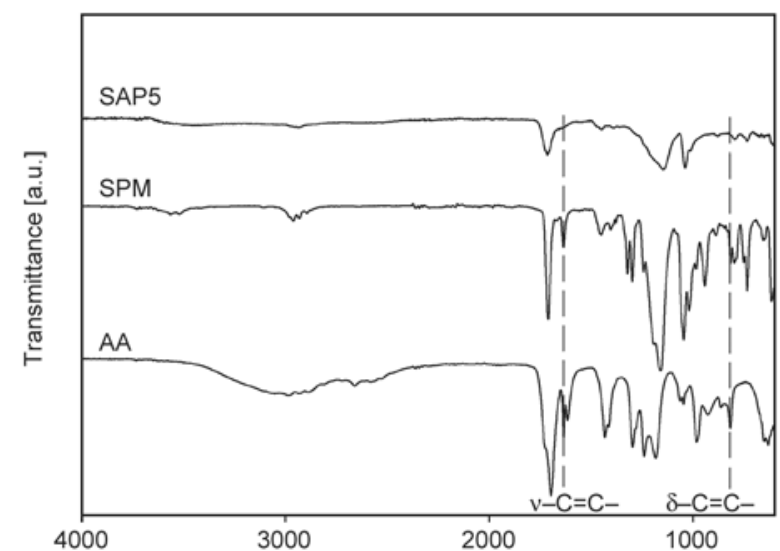

a)

Wave number $\left[\mathrm{cm}^{-1}\right]$

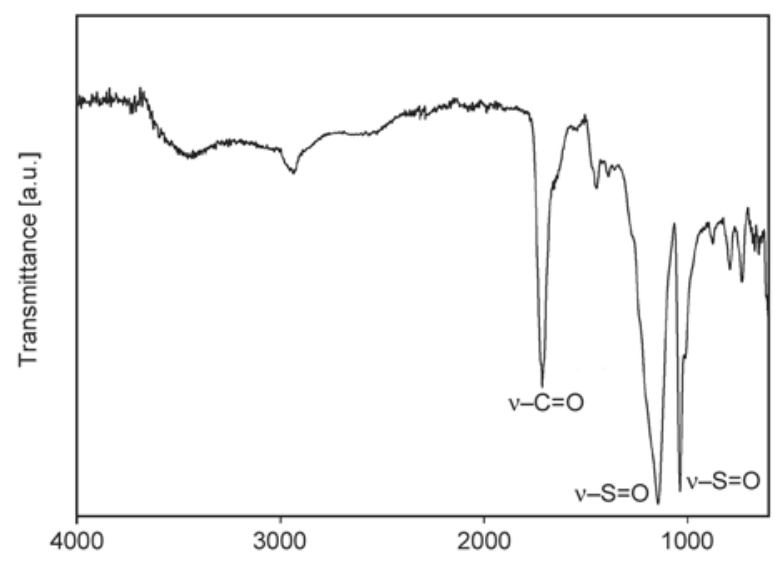

b)

Wave number $\left[\mathrm{cm}^{-1}\right]$

Figure 4. FTIR spectra of SAP5, AA and SPM (a) and SAP5 (b)

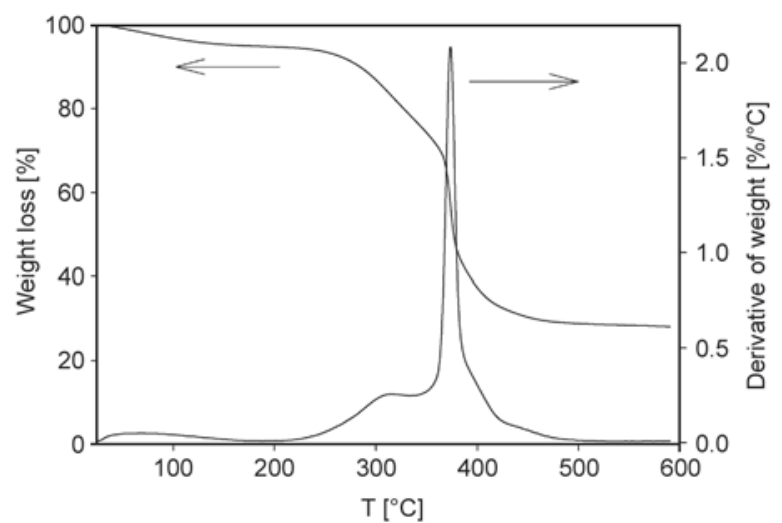

Figure 5. Thermoanalytical curve of SAP5

tributions from both the vibrations of the carbonyl group of ester linkages and from the carbonyl group of the carboxylic acid groups involved in hydrogen bonds. At 1139 and $1038 \mathrm{~cm}^{-1}$ the bands corresponding to the stretching vibrations of the $-\mathrm{S}=\mathrm{O}$ groups appear. The bands corresponding to the stretching

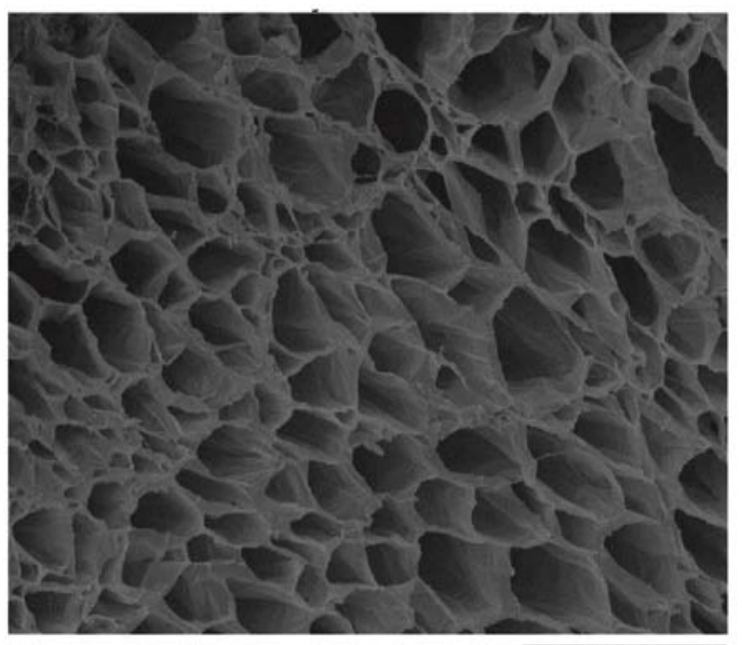

a)

vibrations of the $-\mathrm{P}=\mathrm{O}$ group, that typically appear at $1350-1250 \mathrm{~cm}^{-1}$ are overlapped with those corresponding to the $-\mathrm{S}=\mathrm{O}$ groups [26]. Taking into account the results obtained by the FTIR analysis it is possible to say that the crosslinking reaction was successful.

The thermal stability of SAP5 was evaluated by TGA, from 30 to $600^{\circ} \mathrm{C}$, under a nitrogen flow. Figure 5 gives a global view of the thermal behaviour of SAP5.

It can be observed from Figure 4 that SAP5 undergoes a small initial mass loss of $5 \%$ (close to $100^{\circ} \mathrm{C}$ ), probably due to the existence of residual water in the sample. Around $250^{\circ} \mathrm{C}$, the polymer shows the first relevant and sharp mass loss. This event is due to a degradation of the PAA fractions of the polymer corresponding to the anhydride formation, followed by its decomposition [27]. Finally, the greatest weight loss (approximately $40 \%$ of the total mass)

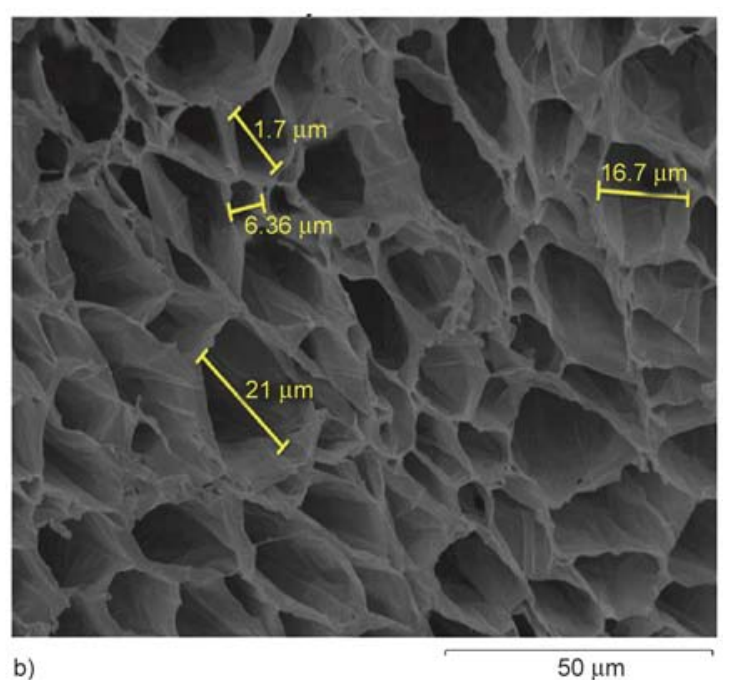

Figure 6. Micrograph of the SAP5 cross-section obtained by SEM: magnification of $750 \times(a)$, and magnification of $1000 \times(b)$ 
from 360 to is close to $450{ }^{\circ} \mathrm{C}$ is due to main chain degradation events [28].

In order to get information about the internal morphology of SAP5, a previously swolen sample was freeze dried and SEM images were taken. Figure 6 presents the images of the SAP5 cross-section obtained by the SEM analysis.

From Figure 6 it is possible to see that SAP5 has a honeycomb like morphology and a high porosity. The highly porous structure presented by SAP5 with pore sizes between 6 and $21 \mu \mathrm{m}$ and with a predominance of large pores allows an easy access of water, which contributes to the good swelling properties of the hydrogel, particularly to the fast kinetics of absorption (Figure 2). Additionally, the great number of pores creates a considerable number of reservoirs for water.

\subsubsection{SAP5 swelling studies in different conditions}

The swelling behaviour of this kind of hydrogels is highly dependent on the ionic strength of the solutions [29]. The effect of the concentration of salt solutions on the swelling behaviour of SAP5 is shown in Figure 7.

As observed in Figure 7, the maximum swelling values for SAP5 in different saline solutions are much lower than those obtained in distilled water, with a reduction of two orders of magnitude, from 2600 to around $60 \mathrm{~g}_{\text {water }} / \mathrm{g}_{\text {dry hydrogel }}(1.5 \mathrm{wt} \% \mathrm{NaCl})$. This result is expected, taking into account the mechanism of swelling of this charged hydrogel and suggests that the developed SAP can be very responsive to salt concentration. As the ionic strength increases, the osmotic pressure, that is the main the

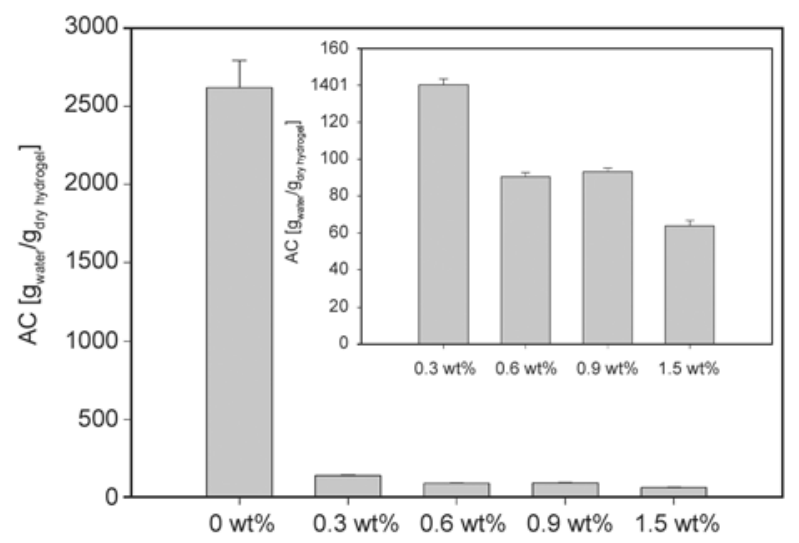

Figure 7. Maximum water absorption capacity of SAP5 in distilled water and in $\mathrm{NaCl}$ solutions with different concentrations, after 24 hours driving force of swelling, is reduced due to a less difference in concentrations of mobile ions between the charged polymer network in the gel and environment solution [30, 31]. Also, as the solution presents high ionic strength, electrostatic repulsion between charged polymer chains is decreased due to shielding effect thus reducing volume availability.

In order to investigate the SAP5 sensibility to the $\mathrm{pH}$, swelling equilibrium experiments were performed to study water absorption at different $\mathrm{pHs}(3,5,7,9$ and 11). The results are presented in Figure 8.

From Figure 8 it is possible to observe that SAP5 exhibits different water absorption values, depending on the $\mathrm{pH}$ of the water solution. The highest water absorption capacity ( $\left.c a .2600 \mathrm{~g}_{\text {water }} / \mathrm{g}_{\text {dry hydrogel }}\right)$ was achieved in neutral medium. It is possible to see that at $\mathrm{pH}$ values lower or higher than 7 , the absorption capacity of the SAP5 is significantly reduced. When SAP5 is in an acidic pH there is an increase in the degree of protonation of the carboxylate (first) and then of the sulfonic groups. With these conditions, the electrostatic repulsion between the negatively charged groups is diminished, causing a contraction of the polymer network [32-34]. The results revealed that for neutral conditions $(\mathrm{pH}=7)$, a maximum number of ionized carboxylate and sulfonate groups are achieved and the electrostatic repulsive force between them is at its highest value, contributing to the great water absorption capacity shown by the SAP sample. At $\mathrm{pH}$ higher than 7, the increase in the concentration of the sodium cations exerts a screening effect over the anions and the amount of ions inside the network also increases. These effects prevent the repulsive forces between the polymeric charged chains and increases the ionic strength of the solution

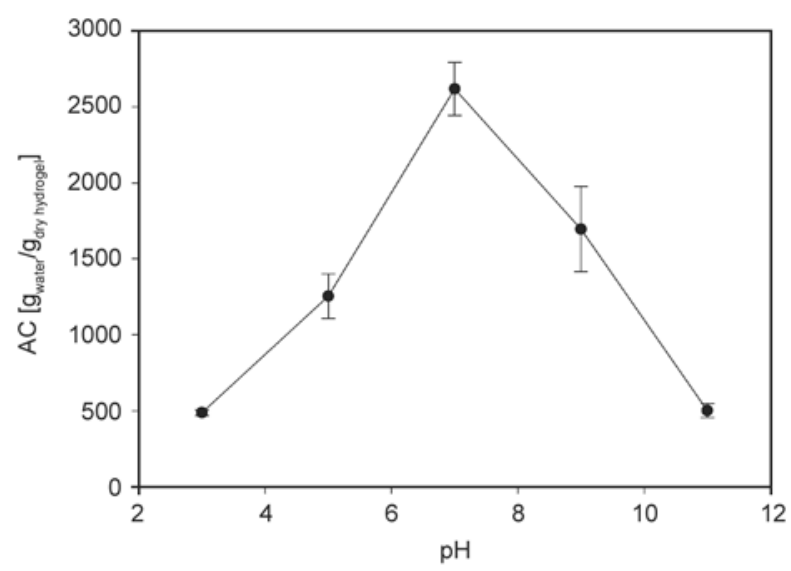

Figure 8. Water absorption capacity of SAP5 at different pHs, after 24 hours 
near polymer surfaces, reducing the total swelling capacity [28].

\subsubsection{Water retention in mixture soil/SAP5}

For agricultural use it is important to evaluate the ability of the hydrogel to retain water in the soil, as well as the benefits of this material for the improvements in soil water absorption capacity. Figure 9 shows the percentage of water retained by the soil for several days after an initial irrigation of a soil sample ( $50 \%$ of water relatively to initial soil mass) with different amounts of SAP5 [15]. The value of $100 \%$ corresponds to the initial weight of water/ soil/polymer mixtures.

From Figure 9, it can be seen that the amount of water in the control soil (without SAP) reaches zero after 11 days. In the soil with $0.5 \mathrm{wt} \%$ SAP the amount of water only reaches $0 \%$ between 12 and 14 days. In soils with 1 and $2 \mathrm{wt} \%$ of SAP, there is still water present in the soil even after 15 days. These results show the clear role of SAP 5 in the improvement of soil humidity which results in a gain of $50 \%$ of time to reach soil dryness. During the first 2 days of the test the curves have a similar profile, which indicates that the water being lost corresponds to water bonded to the soil. Near day 3, the slope of the curves diverges according to the amount of SAP present in the mixture. This observation indicates that, beyond this time, the water loss corresponds to water bonded to the hydrogel $[15,16]$.

\subsubsection{SAP 5 urea release ability}

In addition to the water absorption properties, superabsorbent hydrogels could also be useful in the con-

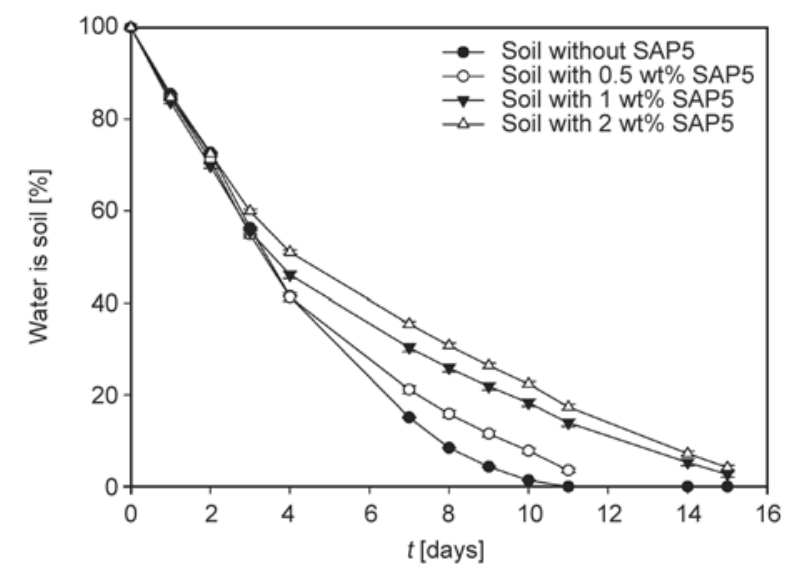

Figure 9. Water retention in soil and in mixtures soil/SAP5. Control soil without any SAP (blank) and soils with increasing amount of $\operatorname{SAP}(0.5,1$ and $2 \mathrm{wt} \%)$. trolled release of fertilizers and particularly urea, one of the most frequently used compounds [35]. First, the hydrogel's capacity to load urea was studied using urea solutions with different concentrations. After promoting the swelling of SAP5 in these solutions, the hydrogels were freeze dried and, by measuring their weights, it was possible to calculate the amount of loaded urea (Figure 10).

As expected, the loading capacity increases for higher amounts of urea available in the solution, due to a higher diffusion to the hydrogel structure. SAP5 shows a urea loading capacity that reaches the maximum value of $30 \mathrm{~g}_{\text {of urea }} / \mathrm{g}_{\text {polymer }}$ (corresponding to $3000 \%$ ) when in contact with a urea solution with $20 \mathrm{~g} / \mathrm{L}$ of concentration. This value of loading capacity is significantly higher than those presented in literature for agricultural hydrogels [14], and is a direct consequence of the high absorption capacity of SAP5.

Also important is the ability of the hydrogel to release the urea. The dry hydrogel obtained in the experiment with the urea solution of $20 \mathrm{~g} / \mathrm{L}$ was placed in distilled water and the amount of urea released with time was monitored gravimetrically. The results are shown in Figure 11.

In terms of urea release, it can be seen from Figure 11 that the process is quite slow, especially when compared with other works reported [14]. After 8 hours, only $50 \%$ of the absorbed urea was released, and only after 48 hours did the release achieve a plateau, corresponding to the release of $91 \%$ of urea originally present in the SAP. Taking into account the fast kinetics of water absorption (see Figure 3), a faster release of the urea from the SAP was expected. However, it is known that urea can interact with carboxylic acid groups by hydrogen bonding [36,

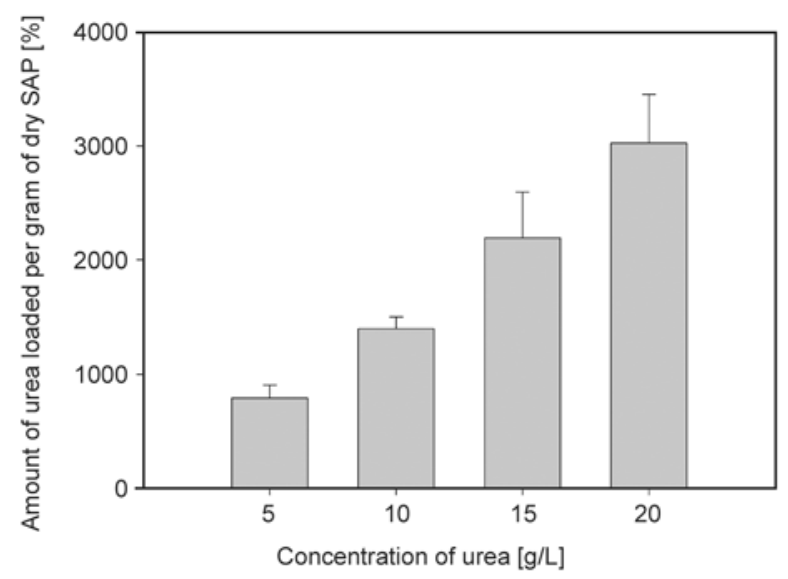

Figure 10. Effect of urea solution on the loading [\%] of SAP5 


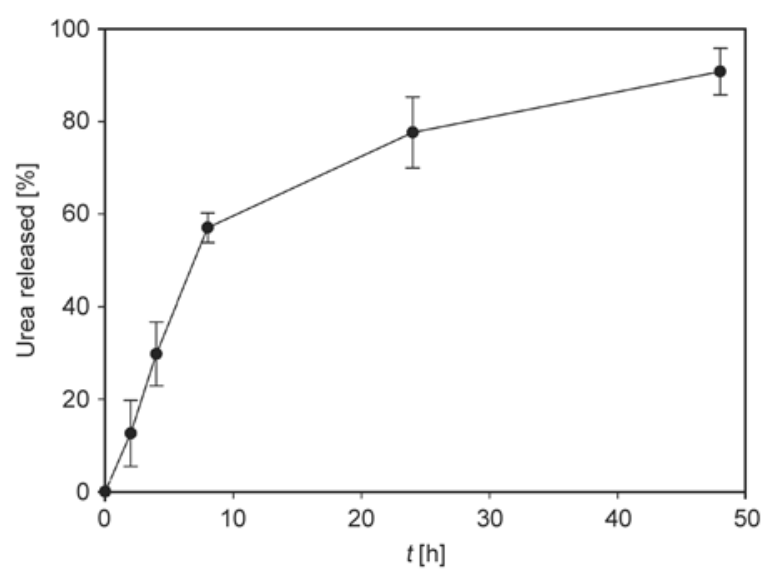

Figure 11. Urea release profile of urea loaded SAP5

37], a fact that might contribute to a slower urea release.

\section{Conclusions}

A set of novel SAPs based on AA, KA, SPM and NIPAM monomers, and BMEP bifunctional crosslinker, were prepared by free radical polymerization, using KPS as initiator. Several formulations were tested and the best results were obtained for a SAP (SAP5) made from AA (1,270 M), SPM (0,715 M), with a concentration of BMEP of $0,00118 \mathrm{M}$. SAP5 presents excellent water absorption capacity ( $\left.2618 \mathrm{~g}_{\text {water }} / \mathrm{g}_{\text {dry hydrogel }}\right)$ when compared to the commonly reported hydrogels. It has a highly porous structure and good thermal stability. Moreover, when mixed with the soil it allows improvements in water retention. This hydrogel has the capacity of being loaded with high amounts of urea ( $\left.30 \mathrm{~g} / \mathrm{g}_{\text {dry hydrogel }}\right)$ and presents a slow urea release when placed in water. To sum up, the results obtained in this work indicate that, by its characteristics, SAP5 can be a potential candidate for agricultural applications.

\section{Acknowledgements}

This work was developed during the Agrofoam project in collaboration with Flex2000 and the authors gratefully acknowledge Qren Program 34022 for financial support.

The NMR data was collected at the UC-NMR facility which is supported in part by FEDER - European Regional Development Fund through the COMPETE Programme (Operational Programme for Competitiveness) and by National Funds through FCT - Fundação para a Ciência e a Tecnologia (Portuguese Foundation for Science and Technology) through grants REEQ/481/QUI/2006, RECI/QEQ-QFI/ 0168/2012, CENTRO-07-CT62-FEDER-002012, and Rede Nacional de Ressonância Magnética Nuclear (RNRMN).

\section{References}

[1] Hüttermann A., Orikiriza L. J. B., Agaba H.: Application of superabsorbent polymers for improving the ecological chemistry of degraded or polluted lands. CLEAN - Soil, Air, Water, 37, 517-526 (2009). DOI: 10.1002/clen.200900048

[2] Ahmed E. M.: Hydrogel: Preparation, characterization, and applications: A review. Journal of Advanced Research, 6, 105-121 (2015). DOI: $10.1016 /$ j.jare.2013.07.006

[3] Zohuriaan-Mehr M. J., Kabiri K.: Superabsorbent polymer materials: A review. Iranian Polymer Journal, 17, 451-477 (2008).

[4] Laftah W. A., Hashim S., Ibrahim A. N.: Polymer hydrogels: A review. Polymer-Plastics Technology and Engineering, 50, 1475-1486 (2011). DOI: $10.1080 / 03602559.2011 .593082$

[5] Sannino A., Demitri C., Madaghiele M.: Biodegradable cellulose-based hydrogels: Design and applications. Materials, 2, 353-373 (2009). DOI: $10.3390 / \mathrm{ma} 2020353$

[6] Döll P.: Impact of climate change and variability on irrigation requirements: A global perspective. Climatic Change, 54, 269-293 (2002).

DOI: 10.1023/A:1016124032231

[7] Dabhi R., Bhatt N., Pandit B.: Super absorbent polymers - An innovative water saving technique for optimizing crop yield. International Journal of Innovative Research in Science, Engineering and Technology, 2, 5333-5340 (2013).

[8] Zohuriaan-Mehr M. J., Omidian H., Doroudiani S., Kabiri K.: Advances in non-hygienic applications of superabsorbent hydrogel materials. Journal of Materials Science, 45, 5711-5735 (2010).

DOI: $10.1007 / \mathrm{s} 10853-010-4780-1$

[9] Bakass M., Mokhlisse A., Lallemant M.: Absorption and desorption of liquid water by a superabsorbent polymer: Effect of polymer in the drying of the soil and the quality of certain plants. Journal of Applied Polymer Science, 83, 234-243 (2002).

DOI: 10.1002/app.2239

[10] Bertoldo M., Bronco S., Gragnoli T., Ciardelli F.: Modification of gelatin by reaction with 1,6-diisocyanatohexane. Macromolecular Bioscience, 7, 328-338 (2007). DOI: $10.1002 / \mathrm{mabi} .200600215$

[11] Venkatachalam D., Vediappan V., Kaliappa Gounder S.: Synthesis and evaluation of trimethylolpropane triacrylate crosslinked superabsorbent polymers for conserving water and fertilizers. Journal of Applied Polymer Science, 129, 1350-1361 (2013).

DOI: $10.1002 / a p p .38826$

[12] Flory P. J., Rehner J.: Statistical mechanics of crosslinked polymer networks II. Swelling. The Journal of Chemical Physics, 11, 521-526 (1943).

DOI: $10.1063 / 1.1723792$

[13] Yarimkaya S., Basan H.: Synthesis and swelling behavior of acrylate-based hydrogels. Journal of Macromolecular Science Part A, 44, 699-706 (2007). DOI: $\underline{10.1080 / 10601320701351268}$ 
[14] Raafat A. I., Eid M., El-Arnaouty M. B.: Radiation synthesis of superabsorbent CMC based hydrogels for agriculture applications. Nuclear Instruments and Methods in Physics Research Section B: Beam Interactions with Materials and Atoms, 283, 71-76 (2012).

DOI: 10.1016/j.nimb.2012.04.011

[15] Abd El-Rehim H. A.: Characterization and possible agricultural application of polyacrylamide/sodium alginate crosslinked hydrogels prepared by ionizing radiation. Journal of Applied Polymer Science, 101, 35723580 (2006).

DOI: $10.1002 / a p p .22487$

[16] Demitri C., Scalera F., Madaghiele M., Sannino A., Maffezzoli A.: Potential of cellulose-based superabsorbent hydrogels as water reservoir in agriculture. International Journal of Polymer Science, 2013, 435073/1435073/6 (2013).

DOI: $10.1155 / 2013 / 435073$

[17] Jang J., Jeong Y-K.: Synthesis and flame-retardancy of UV-curable methacryloyloxy ethyl phosphates. Fibers and Polymers, 9, 667-673 (2008).

DOI: $10.1007 / \mathrm{s} 12221-008-0105-2$

[18] Okay O.: General properties of hydrogels. in 'Hydrogels sensors and actuators' (eds.: Gerlach G., Arndt KF.) Springer, Berlin, 1-14 (2010).

[19] Hunkeler D.: Mechanism and kinetics of the persulfateinitiated polymerization of acrylamide. Macromolecules, 24, 2160-2171 (1991).

DOI: $10.1021 / \mathrm{ma} 00009 \mathrm{a} 004$

[20] Subramanian K.: Photodecomposition of poly(styrene peroxide) in vinyl monomers and structural features of the formed polymers. European Polymer Journal, 37, 55-64 (2001).

DOI: 10.1016/S0014-3057(00)00076-8

[21] Stevens M. P.: Polymer chemistry: An introduction. Oxford University Press, Oxford (1999).

[22] Peng G., Xu S., Peng Y., Wang J., Zheng L.: A new amphoteric superabsorbent hydrogel based on sodium starch sulfate. Bioresource Technology, 99, 444-447 (2008).

DOI: 10.1016/j.biortech.2007.01.018

[23] Scognamillo S., Alzari V., Nuvoli D., Illescas J., Marceddu S., Mariani A.: Thermoresponsive super water absorbent hydrogels prepared by frontal polymerization of $N$-isopropyl acrylamide and 3-sulfopropyl acrylate potassium salt. Journal of Polymer Science Part A: Polymer Chemistry, 49, 1228-1234 (2011).

DOI: $10.1002 /$ pola. 24542

[24] Elliot M.: Superabsorbent hydrogels. BASF report (2014).

[25] Karadağ E., Saraydin D.: Swelling of superabsorbent acrylamide/sodium acrylate hydrogels prepared using multifunctional crosslinkers. Turkish Journal of Chemistry, 26, 863-875 (2002).

[26] Coates J.: Interpretation of infrared spectra, A practical approach. in 'Encyclopedia of analytical chemistry' (ed.: Meyers R. A.) Wiley, Chichester (2000).

DOI: $10.1002 / 9780470027318 . a 5606$
[27] Garay M. T., Alava C., Rodriguez M.: Study of polymer-polymer complexes and blends of poly $(N$-isopropylacrylamide) with poly(carboxylic acid). 2. Poly (acrylic acid) and poly(methacrylic acid) partially neutralized. Polymer, 41, 5799-5807 (2000).

DOI: 10.1016/S0032-3861(99)00765-X

[28] Chen Y., Tan H-M.: Crosslinked carboxymethylchitosan-g-poly(acrylic acid) copolymer as a novel superabsorbent polymer. Carbohydrate Research, 341, 887896 (2006).

DOI: $10.1016 /$ j.carres.2006.01.027

[29] Quesada-Pérez M., Maroto-Centeno J. A., Forcada J., Hidalgo-Alvarez R.: Gel swelling theories: The classical formalism and recent approaches. Soft Matter, 7, 10536-10547 (2011).

DOI: $10.1039 / \mathrm{C} 1 \mathrm{SM} 06031 \mathrm{G}$

[30] Khare A. R., Peppas N. A.: Swelling/deswelling of anionic copolymer gels. Biomaterials, 16, 559-567 (1995) DOI: 10.1016/0142-9612(95)91130-Q

[31] Liu X., Tong Z., Hu O.: Swelling equilibria of hydrogels with sulfonate groups in water and in aqueous salt solutions. Macromolecules, 28, 3813-3817 (1995).

DOI: $10.1021 / \mathrm{ma} 00115 \mathrm{a} 010$

[32] Chen J., Zhao Y.: An efficient preparation method for superabsorbent polymers. Journal of Applied Polymer Science, 74, 119-124 (1999).

DOI: 10.1002/(SICI)1097-4628(19991003)74:1<119::

\section{AID-APP14>3.0.CO;2-T}

[33] Bao Y., Ma J., Li N.: Synthesis and swelling behaviors of sodium carboxymethyl cellulose-g-poly(AA-co-AMco-AMPS)/MMT superabsorbent hydrogel. Carbohydrate Polymers, 84, 76-82 (2011).

DOI: $10.1016 /$ j.carbpol.2010.10.061

[34] Mahdavinia G. R., Pourjavadi A., Hosseinzadeh H., Zohuriaan M. J.: Modified chitosan 4. Superabsorbent hydrogels from poly(acrylic acid-co-acrylamide) grafted chitosan with salt- and $\mathrm{pH}$-responsiveness properties. European Polymer Journal, 40, 1399-1407 (2004). DOI: 10.1016/j.eurpolymj.2004.01.039

[35] Zheng T., Liang Y., Ye S., He Z.: Superabsorbent hydrogels as carriers for the controlled-release of urea: Experiments and a mathematical model describing the release rate. Biosystems Engineering, 102, 44-50 (2009).

DOI: 10.1016/j.biosystemseng.2008.09.027

[36] Jones A. O. F., Leech C. K., McIntyre G. J., Wilson C. C., Thomas L. H.: Engineering short, strong hydrogen bonds in urea di-carboxylic acid complexes. CrystEngComm, 16, 8177-8184 (2014). DOI: $10.1039 / \mathrm{C} 4 \mathrm{CE} 00587 \mathrm{~B}$

[37] Ohlam R., Narasimhan B., Ohlan S., Narang R., Judge V.: Synthesis and antimicrobial evaluation of urea inclusion complexes. Organic Communications, 1, 2432 (2008). 